\title{
Estimation of Individual Muscular Forces of the Lower Limb during Walking Using a Wearable Sensor System
}

\author{
Suin Kim, Kyongkwan Ro, and Joonbum Bae \\ Department of Mechanical Engineering, UNIST, Ulsan, Republic of Korea \\ Correspondence should be addressed to Joonbum Bae; jbbae@unist.ac.kr
}

Received 28 October 2016; Revised 24 February 2017; Accepted 26 March 2017; Published 22 May 2017

Academic Editor: Jaime Lloret

Copyright ( 2017 Suin Kim et al. This is an open access article distributed under the Creative Commons Attribution License, which permits unrestricted use, distribution, and reproduction in any medium, provided the original work is properly cited.

\begin{abstract}
Although various kinds of methodologies have been suggested to estimate individual muscular forces, many of them require a costly measurement system accompanied by complex preprocessing and postprocessing procedures. In this research, a simple wearable sensor system was developed, combined with the inverse dynamics-based static optimization method. The suggested method can be set up easily and can immediately convert motion information into muscular forces. The proposed sensor system consisted of the four inertial measurement units (IMUs) and manually developed ground reaction force sensor to measure the joint angles and ground reaction forces, respectively. To verify performance, the measured data was compared with that of the camera-based motion capture system and a force plate. Based on the motion data, muscular efforts were estimated in the nine muscle groups in the lower extremity using the inverse dynamics-based static optimization. The estimated muscular forces were qualitatively analyzed in the perspective of gait functions and compared with the electromyography signal.
\end{abstract}

\section{Introduction}

Many studies have been conducted to estimate muscular forces in living things, especially humans. To understand the mechanism of the human body performing a motor task quantitatively, it is essential to estimate muscular forces, which has many potential applications: (1) fundamental and quantitative analysis of human motion [1], (2) design of systemic training and analysis of injury mechanisms in the field of sports science [2], (3) diagnosis of abnormal muscular functions and evaluation of the therapeutic effects of rehabilitation [3], and (4) design and development of prosthetic and orthotic [4] and human-robot interaction systems such as an exoskeleton [5-7].

However, although various methodologies have been proposed, estimation of muscular forces remains as a challenging task. Among them, the most simple is to connect a force transducer directly to a muscle [8]. However, it cannot be applied to the human because it requires surgery to the body. Surface electromyography (sEMG) is the most widely accepted noninvasive method to measure the activation level of a muscle [9]. However, it requires complex and frequent calibration processes due to the high noise/signal ratio, differences among individuals, and surrounding conditions. Additionally, only superficial muscles can be measured and it is difficult to block interference from neighboring muscles. Moreover, the true relationship between EMG signals and muscular forces is not yet fully understood. Other researchers have attempted to measure muscle hardness using ultrasound waves and muscle volume using strain sensors, but these have similar drawbacks to EMG $[10,11]$.

Recently, computational simulation programs, such as OpenSim [12], were developed to analyze motor tasks. They provide a model-based estimation method, which enables to build a musculoskeletal model and analyze movements and muscle functions conveniently. The fundamental algorithm within the simulation is "computed muscle control" (CMC), which aims to find the optimal muscle activation according to given kinematic data of the body, such as joint angles, velocities, and acceleration [13]. The algorithm demands relatively high computational costs due to the intrinsic calculation processes including both optimization and forward dynamics.

The inverse dynamics-based static optimization is one of model-based estimation methods, which requires little computation cost, because it does not include a forward 
dynamic process. In addition, unlike the other model-based estimation methods, it is possible to be implemented in realtime, since the inverse dynamics-based static optimization does not demand the full set of motion data in advance. The inverse dynamics-based static optimization has been applied to various motor tasks: walking [14-21], spinal compression [22-27], finger [28], wrist [29], elbow [30,31], arm, and shoulder [32-34] movements.

More specifically, the first step in the inverse dynamicsbased static optimization is to capture the motions and external forces applied to the body. In previous research, the body movements were usually measured by a camera-based motion capture system, and external forces were measured by a force plate. Because the measurement systems have inherent limitations in terms of mobility, this is only available within a laboratory environment and natural movement may be restricted by use of a fixed force plate. In addition, it is not easy to equip the experimental instruments because of spatial and cost limitations.

To overcome the limitations, the camera-based motion capture system has been replaced with the inertial measurement units (IMUs). Recently, full body motion capture systems using the IMUs have been developed [35-39], and there are several commercialized products by Xsens [40], Perception Neuron [41], Synertial [42], Meta Motion [43], Technaid [44], and so on. Using the sensor system, it has been tried to estimate biomechanical factors such as joint moments of the lower limb [45-47]. However, the previous researches have not focused on combining the mobile motion capture system and a shoe-type ground reaction force sensor to investigate individual muscular efforts.

Therefore, in this study, the muscular forces are investigated by integrating a simple wearable sensor system and the inverse dynamics-based static optimization. The wearable sensor consists of four IMUs and an insole-type ground reaction force (GRF) sensor. Analogous to the proposed sensor system, the wireless M3D gait analysis system was developed by Tec Gihan and Human Dynamics Analysis (HDA) was also developed by Insenco [46]. The two systems are composed of a number of IMUs and force transducers attached below the shoes. Even though a rigid force transducer usually allows great accuracy of force measurement and multiaxial application, the user's natural motion can be disturbed due to rigidity and weight of the sensor. The foot plantar pressure sensor should be thin and flexible and light-weight, less than $300 \mathrm{~g}$, for natural gait of the user $[48,49]$. The proposed GRF sensor was made of silicone tubes covered with fabrics to be light and soft.

Based on the motion information collected by the wearable sensor system, the inverse dynamics-based static optimization method was applied to estimate the individual muscular forces of the lower limb during walking. In our previous work, a prototype of the sensor system was developed and preliminary experimental results were acquired [50]. However, it was difficult to validate the estimated muscular efforts due to uncertainties of the measurement system itself. Therefore, in this study, joint angles and GRF measured by the proposed system are compared with that of vision-based

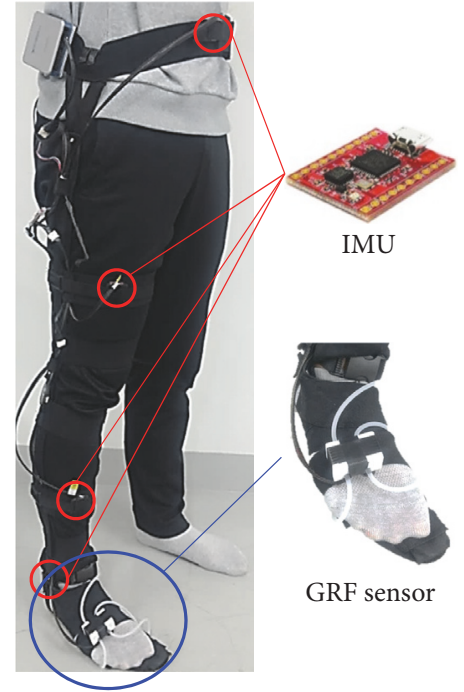

FIGURE 1: Configuration of the wearable sensor system.

motion capture system with a force plate and the estimated muscular efforts were confirmed with the EMG signals.

This paper is organized as follows: detailed procedures for the estimation of individual muscular forces are introduced in the next section, according to the sequence of inverse dynamics-based static optimization. In detail, the inverse dynamic equations of the lower extremity are derived; the required body segment parameters are specified to solve the problems. The static optimization problem is defined based on the musculoskeletal model with selected muscle groups. In the next section, the configuration and functions of the wearable sensor system are described, followed by experimental verification of its performance. In the Results and Discussion, the experimental results are presented, including estimated joint moments and individual muscular forces of the lower limb during normal gait.

\section{Materials and Methods}

2.1. The Wearable Sensor System. The wearable sensor system consists of a wearable structure, four IMUs, and a manually developed insole-type GRF sensor (Figure 1). The objective of the sensor system was to measure the rotational angles of the hip, knee, and ankle joint and two-dimensional pelvic acceleration and GRF, not restraining the motion of the user. For that purpose, the IMU was better than a conventional rotary sensor, such as an encoder, which was required to be aligned with the articular axis. Furthermore, the GRF sensor was fabricated with silicone tubes attached on the insole, which made it lighter and softer than typical force transducers.

2.1.1. Inertial Measurement Unit (IMU). To measure joint angles, the four commercially available 9 DOFs IMUs were adopted, which provide Euler angles [51]. Since the Euler angles were given with respect to the absolute coordinate system (i.e., the earth), the reference frame was defined to 


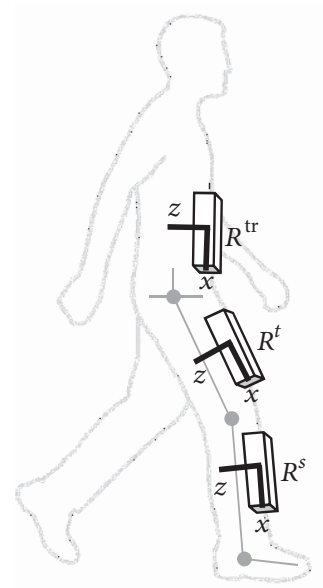

(a)

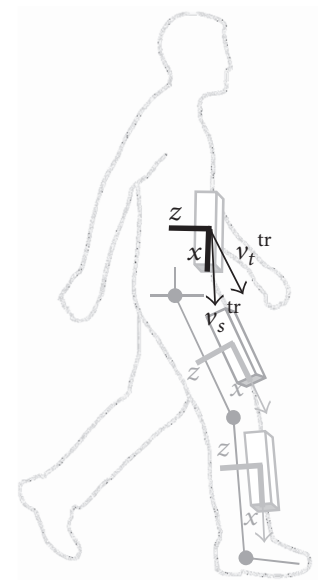

(c)

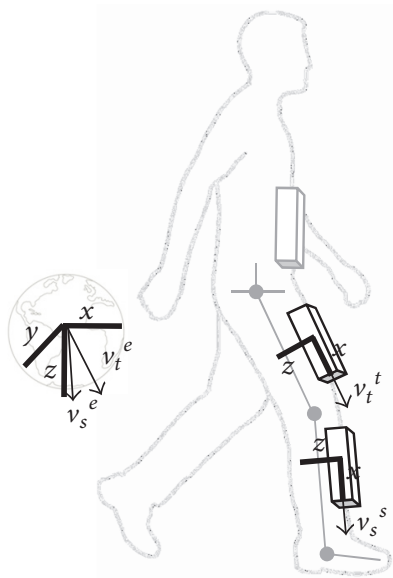

(b)

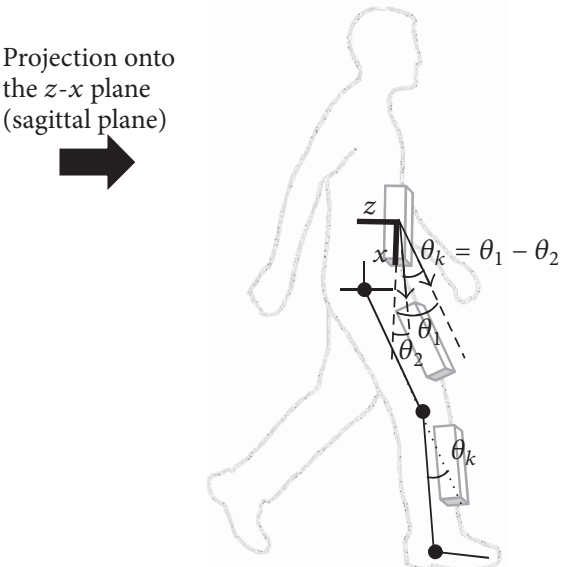

(d)

FIgURE 2: Procedures for converting the Euler angles from the IMUs to the knee joint angle: (a) extracting the rotation matrices from each IMU, (b) calculating the directional vector of each segment, (c) transforming the reference coordinate from the earth to the trunk, and (d) projecting the vectors into the sagittal plane and calculating the joint angle.

measure joint angles by attaching an IMU on the trunk. Therefore, the postures of each segment were calculated as a unit vector with respect to the reference coordinate. For example, the following provides the detailed steps for calculating knee joint angle (Figure 2). The roll axes of the two IMUs attached on the thigh and shank were aligned with the longitudinal axes of each segment (Figure 2(a)), setting the approaching vectors along the longitudinal axis of each IMU:

$$
v_{s}^{s}=\left[(1,0,0)^{T}\right]^{s},
$$

where the superscript indicates the reference coordinate where the vector is expressed and the subscript indicates the approaching vector under consideration out of the thigh, shank, and foot. Consequently, a rotational matrix of the IMU was given as follows:

$$
R=\left[\begin{array}{ccc}
c \gamma c \beta & c \gamma s \beta s \alpha-s \gamma c \alpha & c \gamma s \beta c \alpha+s \gamma s \alpha \\
s \gamma c \beta & s \gamma s \beta s \alpha+c \gamma c \alpha & s \gamma s \beta c \alpha-c \gamma s \alpha \\
-s \beta & c \beta s \alpha & c \beta c \alpha
\end{array}\right],
$$

where $c$ and $s$ are cosine and sine and $\alpha, \beta$, and $\gamma$ are roll, pitch, and yaw angles, respectively. Then, the longitudinal direction of the segment was expressed with respect to the earth by multiplying (2) to (1) (Figure 2(b)):

$$
v_{s}^{e}=\left[\begin{array}{c}
c \gamma c \beta \\
s \gamma c \beta \\
-s \beta
\end{array}\right],
$$

where the superscript $e$ and the subscript $s$ refer to the earth and the segment.

Next, the approaching vectors of the segments can be expressed with respect to the reference coordinate of the IMU on the trunk by multiplying the inverse of (2) on the trunk (Figure 2(c)):

$$
\begin{aligned}
& v_{s}^{\mathrm{tr}} \\
& =\left[\begin{array}{c}
\left(c \beta_{1} c \beta_{2} c\left(\gamma_{1}-\gamma_{2}\right)+s \beta_{1} s \beta_{2}\right) \\
\left(-c \beta_{1} s \beta_{2} s \alpha_{1}+c \beta_{2}\left(c\left(\gamma_{1}-\gamma_{2}\right) s \beta_{1} s \alpha_{1}-c \alpha_{1} s\left(\gamma_{1}-\gamma_{2}\right)\right)\right) \\
\left(-c \beta_{1} c \alpha_{1} s \beta_{2}+c \beta_{2}\left(c \gamma_{1} c\left(\gamma_{1}-\gamma_{2}\right) s \beta_{1}+s \alpha_{1} s\left(\gamma_{1}-\gamma_{2}\right)\right)\right)
\end{array}\right],
\end{aligned}
$$


where the subscript " 1 " refers to the angles of the IMU on the target segment and "2" represents the reference frame on the trunk.

Finally, the direction vectors of the thigh and shank were projected onto the sagittal plane, so that angle of the segments with respect to the body reference frame was calculated using simple trigonometrical functions (Figure 2(d)):

$$
\theta_{i}=-\operatorname{sgn}(a) \arccos \left(\frac{a}{\sqrt{a^{2}+c^{2}}}\right) \quad(i=1,2,3),
$$

where $\theta$ is the angle of the segment with respect to the coordinate of the IMU on the trunk and $a$ and $c$ are the first and third components of (4), respectively. The knee joint angle $\theta_{k}$ was calculated as $\theta_{1}-\theta_{2}$.

An important issue was to align the IMU carefully along the longitudinal direction of the segment; $x z$ plane of each IMU (in Figure 2) was set to be parallel to the sagittal plane of the corresponding segment. To align each IMU, the subject was asked to stand still, and the IMUs were attached on the each segment maintaining the condition that the direction of gravity measured by the IMU was on $x z$ plane. If the condition was satisfied, the $x z$ plane and the sagittal plane could be considered parallel. The IMUs were attached to the wearable structure which was made of straps and rubber bands. The wearable structure hold the IMUs tightly in place to ensure that alignment of IMUs was unchanged during experiments. The initial angles were set to zero before the start of an experiment, by asking the subject to stand and make the leg be straightened. All the same procedures were applied to calculate the joint angles at the hip and ankle.

2.1.2. Ground Reaction Force (GRF) Sensor. The proposed GRF sensor was a modified version of Smart Shoes whose performance was validated in the previous studies [52, 53]. Figure 3 describes its configuration and the weight of the entire insole sensor was $125 \mathrm{~g}$. The GRF sensor only measured force along the vertical axis from the ground by measuring pressure changes within the air bladders. The tangential GRF was assumed to be negligible, because the magnitude of the vertical force is about 20 times more than both the lateral shear force and progressional shear force during walking $[54,55]$. From the force distribution of the four air bladders, the center of pressure (COP) of the GRF was also calculated with respect to the position of the ankle joint.

It was necessary to determine the relationship between the pressure changes within the air bladder and external force applied to it. For the calibration process, while the air bladder was pressurized, the corresponding external force was measured using a force transducer while simultaneously measuring the air pressure within the air bladder. Before the calibration process, to remove hysteresis effect caused by the viscoelastic properties of the silicone tubes, dynamic compensator was applied to compensate dynamic effect of the air bladders. The performance of the dynamic compensator was verified in the previous research $[56,57]$. After the dynamic compensator was applied, the calibration procedures were conducted. The collected results were fitted with a line, as shown in Figure 4, to make a linear model, the slope of

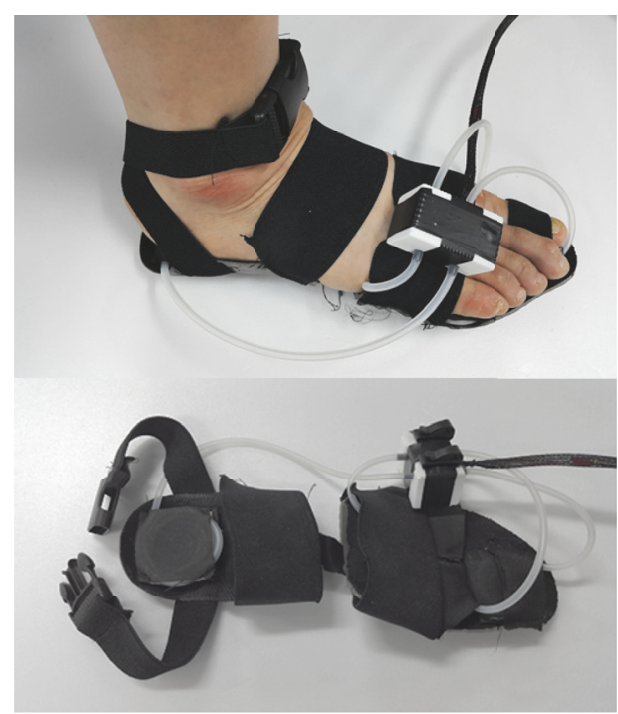

(a)

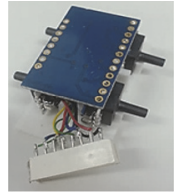

(b)

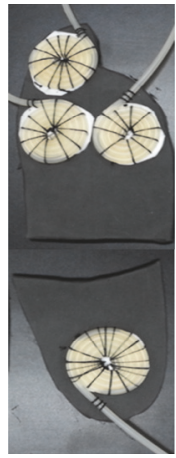

(c)
FIGURE 3: Manually developed GRF sensor with four air bladders: (a) entire view, (b) pressure sensor module, and (c) air bladders attached to the insole.

which was associated with the amount of air inside and the geometry of the bladder. The coefficient of determination, $R^{2}$, was also calculated, which indicated how the acquired data fit a linear model. On the assumption that the slope remained unchanged, it was necessary to initialize the signal to zero for accurate measurements.

2.2. Inverse Dynamics-Based Static Optimization. Individual muscular forces of the lower limb were estimated by applying inverse dynamics-based static optimization through a series of procedures (Table 1). The lower extremity was modeled as a two-dimensional musculoskeletal system in the sagittal plane that includes three segments (thigh, shank, and foot), three joints (hip, knee, and ankle), and nine muscle groups. The model includes information on body segment parameters to solve the inverse dynamics problem and moment arms and the physiological cross-sectional area of each muscle for defining the static optimization problem. Based on the musculoskeletal model, a set of motion data were measured, the motion data were substituted into the inverse dynamic equations to estimate joint moments, and the individual muscular forces were estimated by solving the static optimization based on the joint moments.

2.2.1. Inverse Dynamics. The lower extremity was described as a mechanical system in dynamic equilibrium under the actions of gravity, inertia, ground reactions, and muscular forces, which generate appropriate moments at the joints. Figure 5 shows a free body diagram of the lower extremity in the sagittal plane, considering only flexion/extension motion of the three joints, because most of the movements and forces in the lower limb during walking occur in the plane [58]. The two-dimensional model involves five degrees of freedom, including three joint angles and the planar motion 

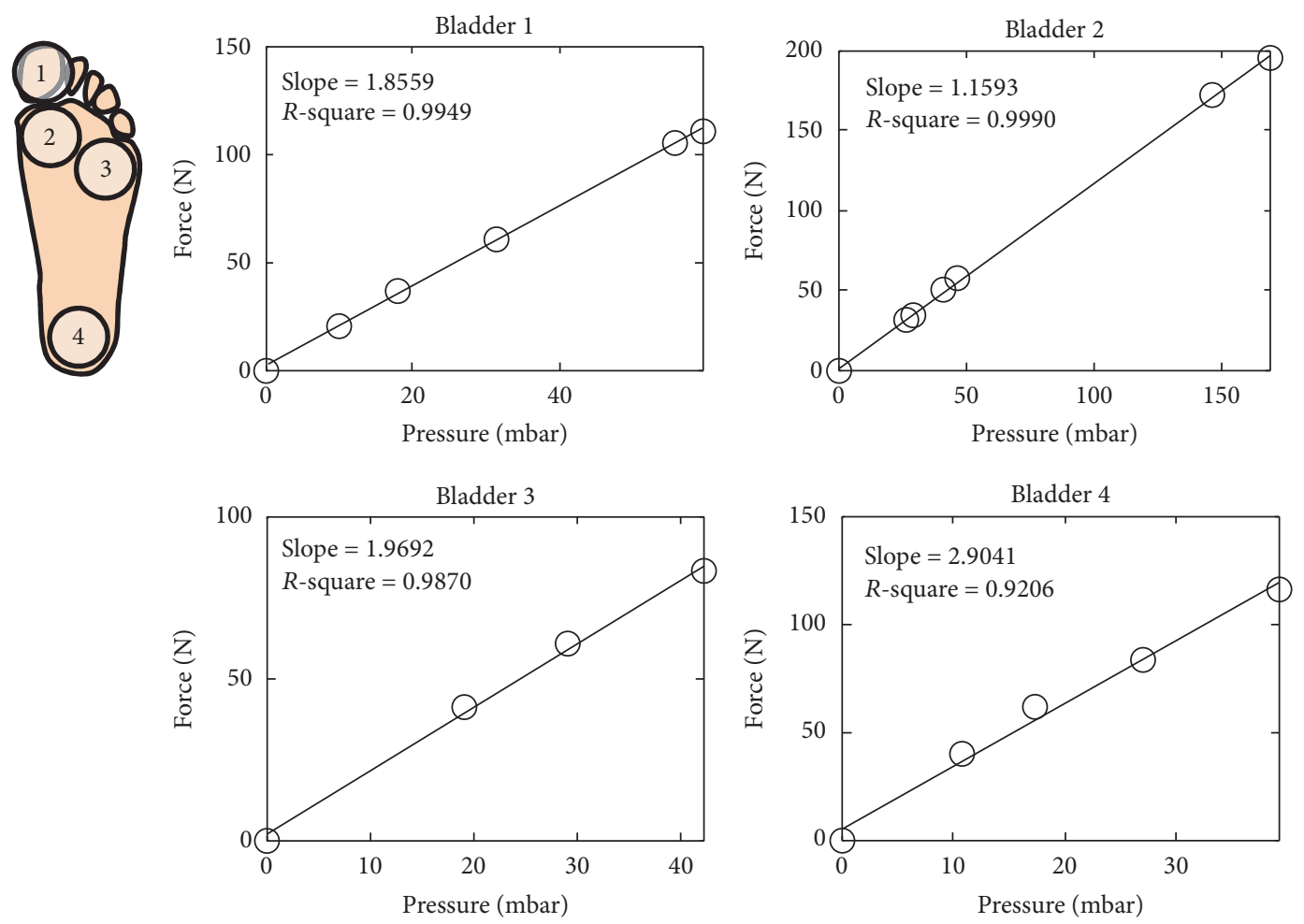

FIGURE 4: Results of the calibration process with the resulting slope and $R^{2}$ value.

of the pelvis. Using Lagrange mechanics, the inverse dynamic equations were derived with respect to the absolute angle of the three segments:

$$
\begin{aligned}
& M_{A}=-F_{\mathrm{GRF}} r \sin \theta_{3}+J_{f} \ddot{\theta}_{3}+g \sin \theta_{3} \\
& +d_{f} m_{f}\left(-l_{t} \dot{\theta}_{1}^{2} \sin \left(\theta_{1}-\theta_{3}\right)+l_{s} \dot{\theta}_{2}^{2} \sin \left(\theta_{2}+\theta_{3}\right)\right. \\
& \quad+l_{t} \ddot{\theta}_{1}^{2} \cos \left(\theta_{1}-\theta_{3}\right)+l_{s} \ddot{\theta}_{2} \cos \left(\theta_{2}+\theta_{3}\right)+d_{f} \ddot{\theta}_{3} \\
& \left.\quad+\ddot{x}_{p} \cos \theta_{3}+\ddot{y}_{p} \sin \theta_{3}\right), \\
& M_{K}=M_{A}+l_{s} F_{\mathrm{GRF}} \sin \theta_{2}-J_{s} \ddot{\theta}_{2}-g\left(l_{s} m_{f}+d_{s} m_{s}\right) \\
& \quad \cdot \sin \theta_{2}-l_{t}\left(l_{s} m_{f}+d_{s} m_{s}\right) \dot{\theta}_{1}^{2} \sin \left(\theta_{1}+\theta_{2}\right) \\
& \quad-d_{f} l_{s} m_{f} \dot{\theta}_{3}^{2} \sin \left(\theta_{2}+\theta_{3}\right)+\left(l_{s} m_{f}+d_{s} m_{s}\right) l_{t} \ddot{\theta}_{1} \\
& \quad \cdot \cos \left(\theta_{1}+\theta_{2}\right)-\left(l_{s}^{2} m_{f}+d_{s}^{2} m_{s}\right) \ddot{\theta}_{2}+d_{f} l_{s} m_{f} \ddot{\theta}_{3} \\
& \quad \cdot \cos \left(\theta_{2}+\theta_{3}\right)+\left(l_{s} m_{f}+d_{s} m_{s}\right) \ddot{x}_{p} \cos \theta_{2}-\left(l_{s} m_{f}\right. \\
& \left.\quad+d_{s} m_{s}\right) \ddot{y}_{p} \sin \theta_{2}, \\
& \quad+M_{H}=M_{K}-l_{t} F_{\mathrm{GRF}} \sin \theta_{2}+J_{t} \ddot{\theta}_{1}+g\left(l_{t}\left(m_{f}+m_{s}\right)\right. \\
& \left.\quad+d_{t} m_{t}\right) \sin \theta_{1}+l_{t}\left(l_{s} m_{f}+d_{s} m_{s}\right) \dot{\theta}_{2}^{2} \sin \left(\theta_{1}+\theta_{2}\right) \\
& +d_{f} l_{t} m_{f} \dot{\theta}_{3}^{2} \sin \left(\theta_{1}-\theta_{3}\right)-l_{s} l_{t} m_{f} \ddot{\theta}_{2} \cos \left(\theta_{1}+\theta_{2}\right)
\end{aligned}
$$

$$
\begin{aligned}
& +\left(l_{t}^{2}\left(m_{f}+m_{s}\right)+d_{t}^{2} m_{t}\right) \ddot{\theta}_{1}-d_{s} l_{t} m_{s} \ddot{\theta}_{2} \cos \left(\theta_{1}+\theta_{2}\right) \\
& +d_{f} l_{t} m_{f} \ddot{\theta}_{3} \cos \left(\theta_{1}-\theta_{3}\right)+\left(l_{t}\left(m_{f}+m_{s}\right)+d_{t} m_{t}\right) \\
& \cdot \ddot{x}_{p} \cos \theta_{1}+\left(l_{t}\left(m_{f}+m_{s}\right)+d_{t} m_{t}\right) \ddot{y}_{p} \sin \theta_{1},
\end{aligned}
$$

where the capital subscripts indicate the relevant joint, hip, knee, and ankle and the small letters represent the segment: pelvis, thigh, shank, and foot. $M$ is the joint moment generated by the muscles, $d$ is the center of mass positions from the proximal joints, $\theta_{1}, \theta_{2}$, and $\theta_{3}$ are the absolute angles of the thigh, shank, and foot, $x$ and $y$ are the center of mass position of each segment, $F_{\mathrm{GRF}}$ is the ground reaction, and $r$ is the application point of the ground reaction from the ankle.

Except for the measured motion data, there exist some required parameters to solve the dynamic equations such as segment length, mass, center of mass position, and mass moment of inertia. However, they are not directly measurable, except for the segment length; thus the other parameters should be estimated using the formulas found in cadaveric experiments [59]:

$$
\begin{aligned}
m & =P \cdot(\text { body mass }), \\
c & =R \cdot(\text { segment length }), \\
I & =m \cdot K \cdot(\text { segment length })^{2},
\end{aligned}
$$

where $m, c$, and $I$ are the mass, the center of mass position, and the mass moment of inertia. The coefficients, $P, R$, and $K$, of each segment are listed in Table $2[60,61]$. 
TABLE 1: Detailed procedures of inverse dynamics-based static optimization.

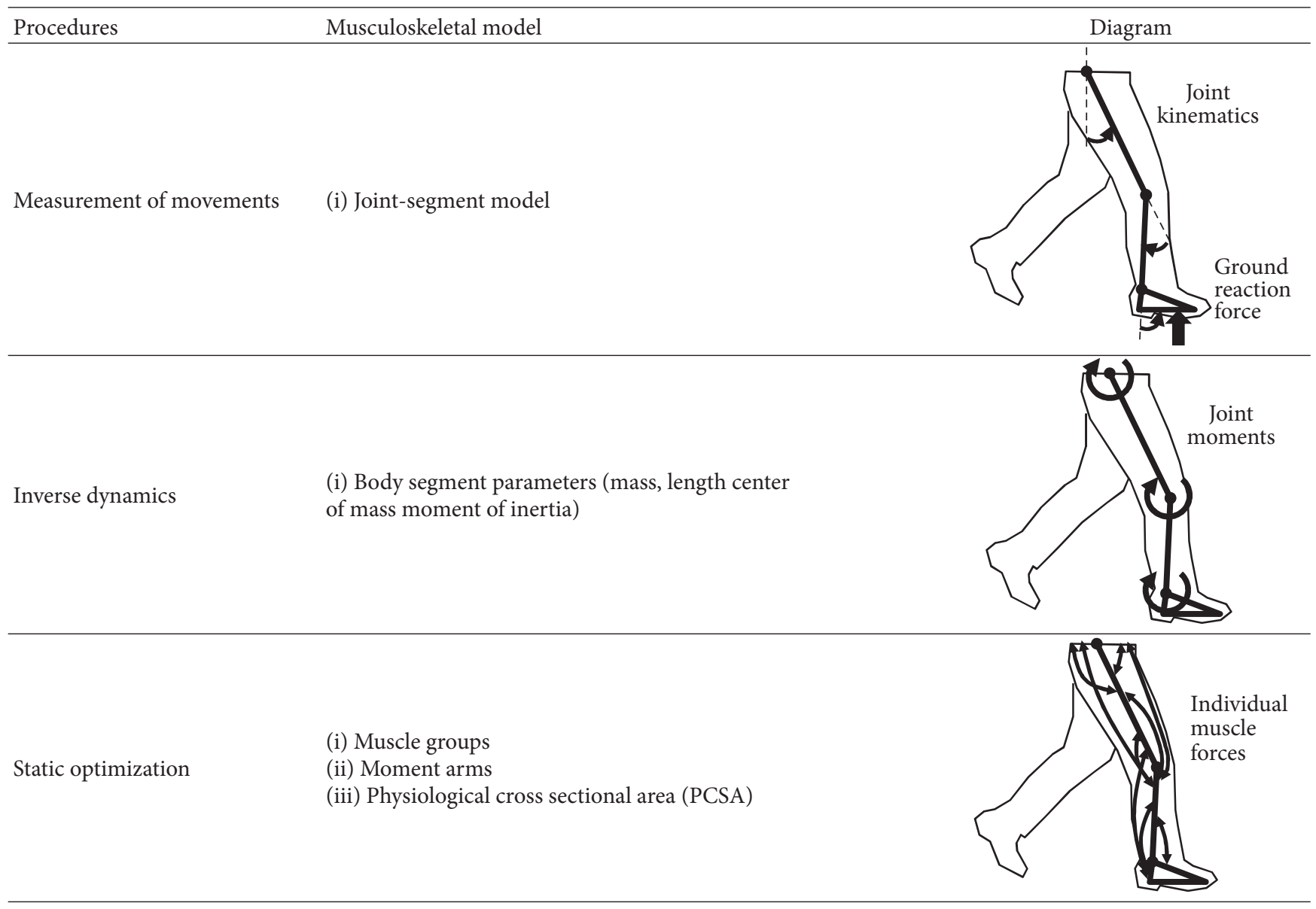

TABLE 2: Coefficients for the body segment parameters.

\begin{tabular}{lccccrr}
\hline & $P$ & $R_{p}$ & $R_{d}$ & $K_{\mathrm{CG}}$ & $K_{p}$ & $K_{d}$ \\
\hline Foot & 0.0145 & 0.500 & 0.500 & 0.475 & 0.690 & 0.690 \\
Leg & 0.0465 & 0.433 & 0.567 & 0.302 & 0.528 & 0.643 \\
Thigh & 0.1000 & 0.433 & 0.567 & 0.323 & 0.540 & 0.653 \\
\hline
\end{tabular}

The subscript means the origin point where the resultant parameter is referring to; $d$ refers to the distal joint, $p$ refers to the proximal joint, and CG refers to the center of gravity $[60,61]$.

2.2.2. Musculoskeletal Model and Static Optimization. Individual muscular forces were determined by the static optimization based on the moment equilibrium equations between net joint moment produced by the muscles and the joint moments calculated from the inverse dynamics. Because the number of muscles is usually larger than that of equilibrium equations, an optimization process is required. The detailed discussion on applying the static optimization is described in this section, including selecting the muscles of interest, calculating moments' arms of each muscle, and choosing an optimization criterion. The optimization problem was solved by a sequential quadratic programming (SQP) algorithm.

As shown in Figure 6, the musculoskeletal model included all of the muscle groups which are functionally independent: (1) the iliacus (IL) for the hip flexor, (2) the hamstrings (HA) for the hip extensor and knee flexor simultaneously, (3) the rectus femoris (RF) for the hip flexor and knee extensor, (4) the gastrocnemius (GA) for the knee flexor and plantar flexor, (5) the biceps femoris short head (BF) for the knee flexor, (6) the vastus (VA) for the knee extensor, (7) the tibialis anterior (TA) for the dorsiflexor, (8) the soleus (SO) for the plantar flexor, and (9) the gluteus maximus (GL) for the hip extensor. The muscles have significant force capabilities and moment arms, such that their contributions to the joint torque are also more significant than the excluded muscles $[62,63]$.

Even though a muscle exerts only a tensile force, it produces torsional force with respect to the joint due to its attachment to the bones. This means that how the muscle 


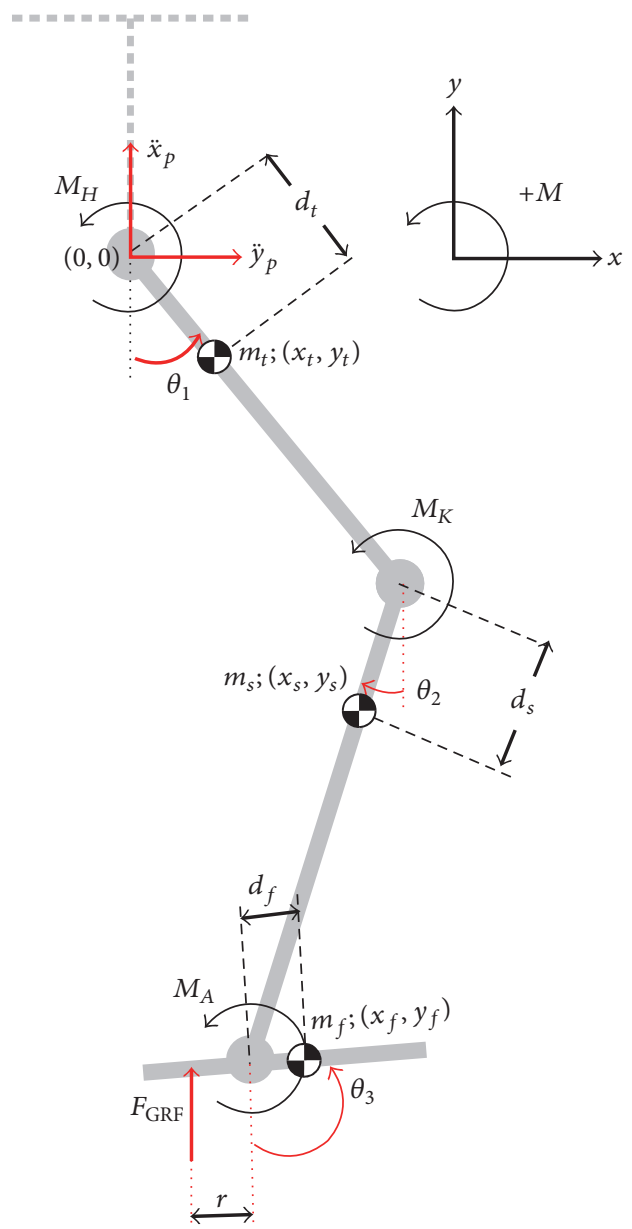

Figure 5: Two-dimensional free body diagram of the lower limb ( $M_{i}$ : joint moment $\left(i=H\right.$ : hip, $K$ : knee, and $A$ : ankle), $\theta_{j}$ : absolute angle of the joints ( $j=1$ : thigh, 2 : shank, and 3 : foot), $\left(x_{k}, y_{k}\right)$ : center of mass position of each segment ( $k=t$ : thigh, $s$ : shank, and $f$ : foot), $F_{\mathrm{GRF}}$ : ground reaction, and $r$ : center of pressure of $\left.F_{\mathrm{GRF}}\right)$.

generates torque about a joint depends on both muscle tendon force and the musculoskeletal geometry. Moment arms of the muscle groups were calculated using the vector operations based on the coordinate data of the muscle origin and insertion with respect to a corresponding reference frame $[64,65]$. For example, Figure 7 illustrates the vector operation to calculate the moment arm of the hamstring, where $v_{o}^{\text {tr }}$ and $v_{i}^{s}$ are the origin and insertion coordinates with respect to reference coordinate, tr is trunk, and $s$ is shank, respectively, given in [65]. Using the same strategy, moment arms of the muscles arms were expressed as a function of joint angles.

To solve the individual muscular forces without reducing the number of muscle groups, an optimization process is required. It is important to adopt the proper optimization criteria, and various types have been suggested to simulate the actual body functions for recruiting the muscles: minimizing (1) muscle force [18], (2) joint moment [14], (3) ligament force, (4) joint contact force, (5) instantaneous muscle power [20], and (6) muscle stress [15]. Among them, the criterion of maximum endurance is the most widely accepted recently,

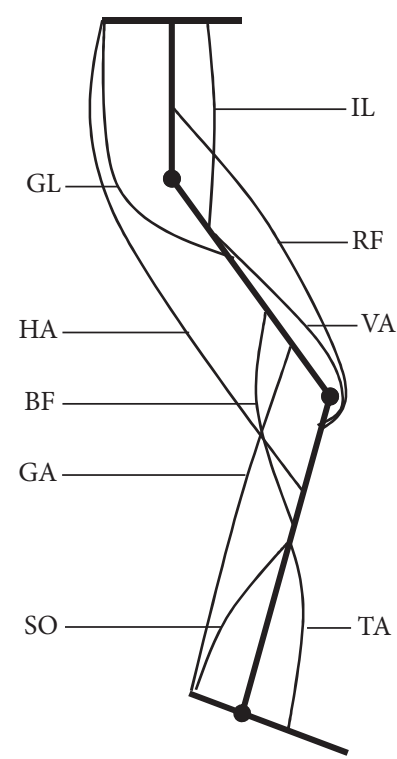

Figure 6: Muscle groups in the sagittal plane (IL: iliacus, HA: hamstrings, RF: rectus femoris, GA: gastrocnemius, BF biceps femoris short head, VA: vastus, TA: tibialis anterior, SO: soleus, and GL: gluteus maximus).

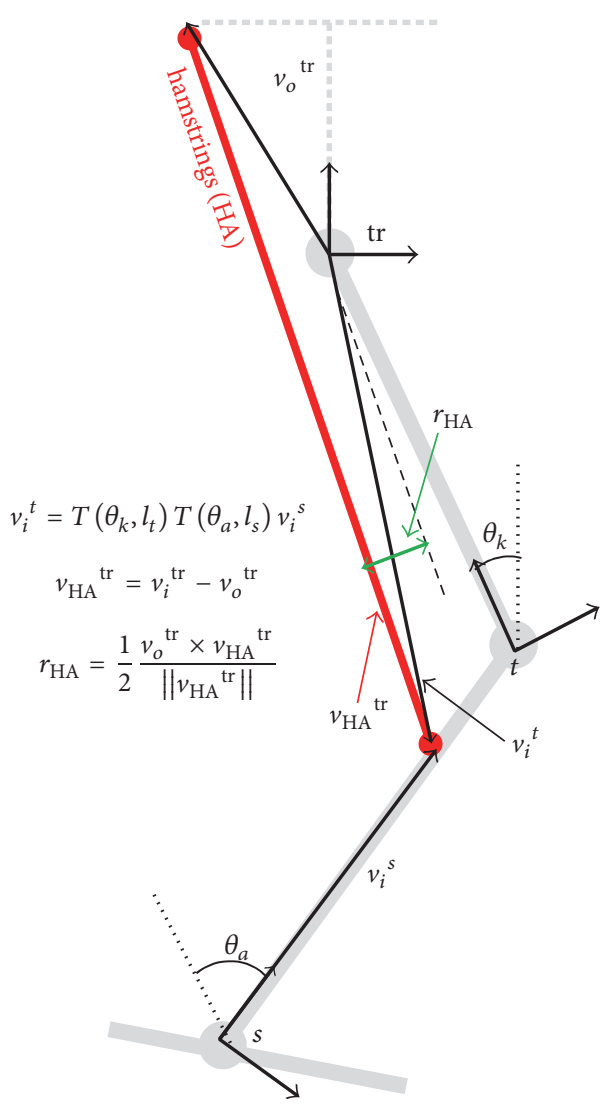

FIgURE 7: Vector operation for calculating moment arm of the hamstring; the subscript identifies the vector $(o, i$ : origin and insertion), the superscript indicates which reference frame the vector is referring to (tr: trunk, $t$ : thigh, and $s$ : shank), $\theta$ : is rotational angle, $l$ is segment length, and $T$ is homogeneous transformation matrix as a function of $\theta$ and $l$. 
because it was demonstrated to be the most biologically meaningful. The cost function to be minimized is as follows:

$$
J=\sqrt[n]{\sum\left(\frac{f_{i}}{A_{i}}\right)^{n}}
$$

where $f_{i}$ is the muscular force and $A_{i}$ is the physiological cross-sectional area (PCSA) of the muscle. The PCSA is defined as the total area of the cross-section perpendicular with respect to the muscle fiber [66], and it is also calculated as the volume of the muscle divided by the optimal fiber length [67]. An appropriate value of the power $n$ in (8) cannot be determined exactly due to differences in individuals and muscles. In addition, to specify the value, it is necessary to measure the muscular forces accurately, which would not be available. Accordingly, the average value in literature reports, $n=3$, has been adopted as the most appropriate value [16].

The optimization problem was specified to minimize the cost function in (8) satisfying the following constraints:

$$
\begin{aligned}
0 & \leq f_{i} \leq\left(f_{i}\right)_{\max }, \\
A X & =B,
\end{aligned}
$$

where $f_{i}$ is the exerted muscular force and $\left(f_{i}\right)_{\max }$ is the maximum isometric force. The first constraint in (9) represents the force capability of each muscle, and the maximum isometric force $\left(f_{i}\right)_{\max }$ is determined by multiplying the PCSA and the specific tension, $61 \mathrm{~N} / \mathrm{cm}^{2}$, of the muscle fiber, which is the maximum stress the fiber can endure. The values of the PCSA in the [67] were used. The other constraint in (10) is moment equilibrium between the joint moment from the inverse dynamics and the resulting moment exerted by the muscles. The detailed representation of (10) is specified in Appendix.

\section{Results and Discussion}

3.1. Verification of the Wearable Sensor System. To verify the proposed wearable sensor system, joint angles and ground reactions were measured during normal walking by the proposed sensor system and camera-based motion capture system (Motion Analysis Corp.) with a force plate (Kistler) simultaneously $[68,69]$. A subject (male, $178 \mathrm{~cm}, 70 \mathrm{~kg}$ ) with no musculoskeletal disease walked at a speed of $3 \mathrm{~km} / \mathrm{h}$. As shown in Figure 8, the joint angles were successfully measured by the proposed sensor system. In addition, preparation time was less than a minute for attachment of the IMUs and initialization of joint angles, which was much faster than the camera-based motion capture system. Ground reactions and its center of pressure were also successfully measured by the proposed sensor system (Figure 9).

3.2. Experimental Results during Walking. Based on the motion data shown in Figures 8 and 9, the joint moments were estimated by solving the inverse dynamics, and the solution of the optimization problem produced instantaneous values of muscular forces in the nine muscle groups at each instant. To interpret the estimated muscular efforts,
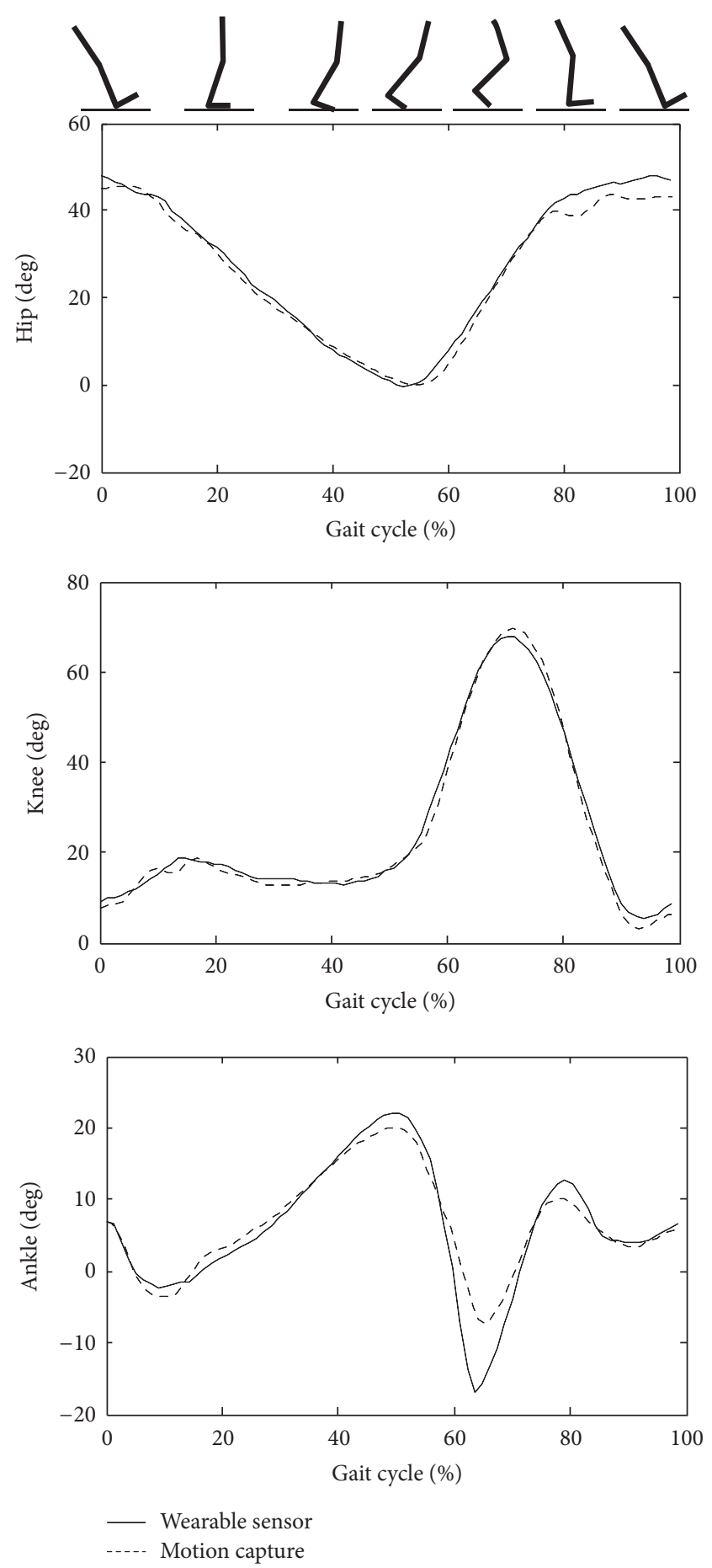

FIGURE 8: Joint angles during normal gait.

the experimental results were organized according to the four important functions of a gait cycle (Figure 10); each phase of gait cycle was identified by the ground reaction patterns in Figure 9.

Heel rocker occurs during the phase of initial contact and the loading response phase, which is required to bear the large flexion torque of the hip joint due to a significant vertical vector (60\% body weight) that is anterior to the hip 

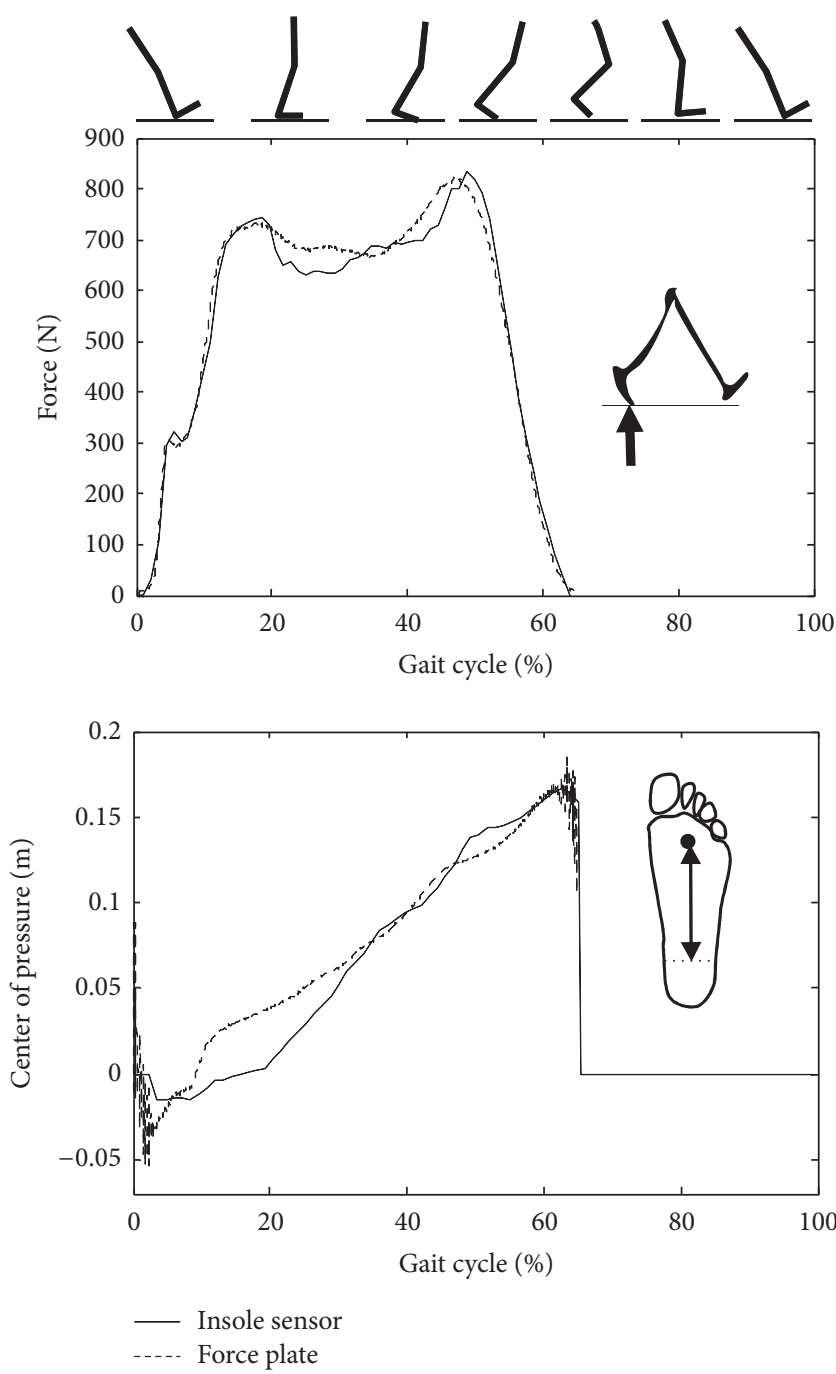

FIGURE 9: Ground reactions and center of pressure position with respect to the ankle position during normal gait.

joint. Thus, in the estimated result, a large extension torque was generated by the hip extensors (HA and GL). Similarly, flexion torque at the knee joint is necessary to endure the vertical ground reaction force. Due to the two-jointed muscle, $\mathrm{HA}$, a large flexion torque was also produced at the knee joint, and it was released by the knee extensor, VA. In the case of the ankle joint, the dorsiflexor TA was excited to endure ground reactions placed behind the ankle. In accordance with the actions of muscles, the shank made its advance (Figure 10(a)).

Ankle rocker occurs during the midstance to continue progression of the body (Figure 10(b)). As the limb rolls forward over the supporting foot, the directions of the joint torques were identically downwards. All of the muscles placed on the posterior side of the limb, such as the GL, HA, BF, GA, and $\mathrm{SO}$, were involved in the motion. The SO muscle, assisted by the GA, was excited to maintain stance stability of the limb. Due to the secured stability gained by the action of the SO and GA muscle, further demand on the quadriceps, including the VA and RF muscle, was minimized.
Forefoot rocker takes place during the terminal stance (Figure 10(c)). The heel rolled over the anchor of the forefoot with the ankle virtually locked by the SO and GA, continuing advancement of the shank. Maximal plantar flexion torque was required because the vertical ground reactions, combined with falling body weight, were applied at the toe. The heel rise posture demanded strong SO and GA action, approximately three times more than in midstance. As the knee began to flex by the end of the terminal stance, the BF muscle was involved in this phase. While the hip was fully extended, the body rolls forward over the forefoot rocker, and the IL and RF muscles were activated as hip flexors to restrain the rate and extent of hip extension.

The limb accomplishes foot clearance in the phase of the initial swing and the midswing (Figure 10(d)). As the shank became vertical, the ankle joint supported the downward force of the foot weight. Thus, the TA muscle was activated at that instant. Because momentum produced by the hip flexion was compensated for the pull of gravity on the shank, joint torque at the knee reached a balance. Additionally, hip flexion of the swing phase was generated by the passive gravitational force of the limb, such that the intervention of the hip and knee muscles was not significant.

The experimental results of individual muscular forces during a normal stride are presented in Figure 11(a). To validate the experimental result, the estimated muscular forces were compared with the another experimental result (Figure 11(b)); muscular forces were estimated by using a model-based estimation algorithm, a camera-based motion capture system and a force plate were used to measure motion information, and EMG signals were also recorded for validation [63]. The estimated muscular forces show similar patterns and magnitudes, and the results in this study coincide with EMG patterns also. Because it is very difficult to measure true muscular forces of a human, it is not easy to validate the estimated muscular forces quantitatively. However, the activation pattern of muscles during normal walking was evidenced by well-documented experimental results with EMG signals [14-16, 19, 20, 54, 70]. Comparing the estimated muscular forces with the electromyography patterns in the previous studies, it was possible to check feasibility of the estimated results.

\section{Conclusions}

Since it is very difficult to measure muscular force directly, model-based estimation method has been researched actively for an alternative. Although there exists the commercialized software package such as OpenSim, the software consists of a complex musculoskeletal model with higher computation costs and technical barrier. Additionally, motions of the human are usually measured camera-based measurement system with a force plate which require time-consuming preparation for attaching reflective markers, data processing procedures, and high cost for space and equipment. Therefore, this study suggests a cost-effective estimation method by integrating the simple sensor system and the algorithm. The proposed sensor system was cost-effective in the aspect of space, equipment expense, preparation, and postprocessing 


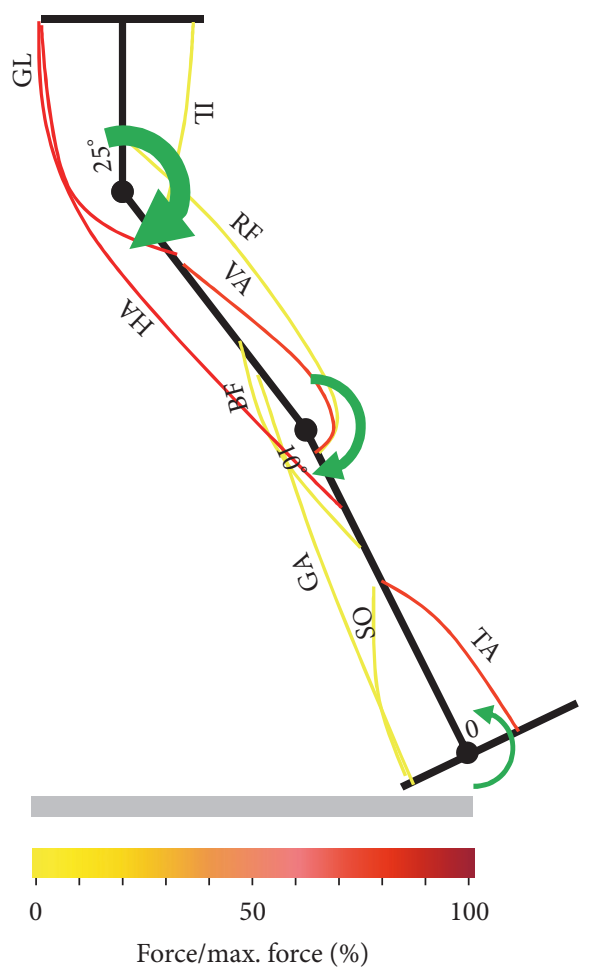

(a) Heel rocker $(0 \%)$

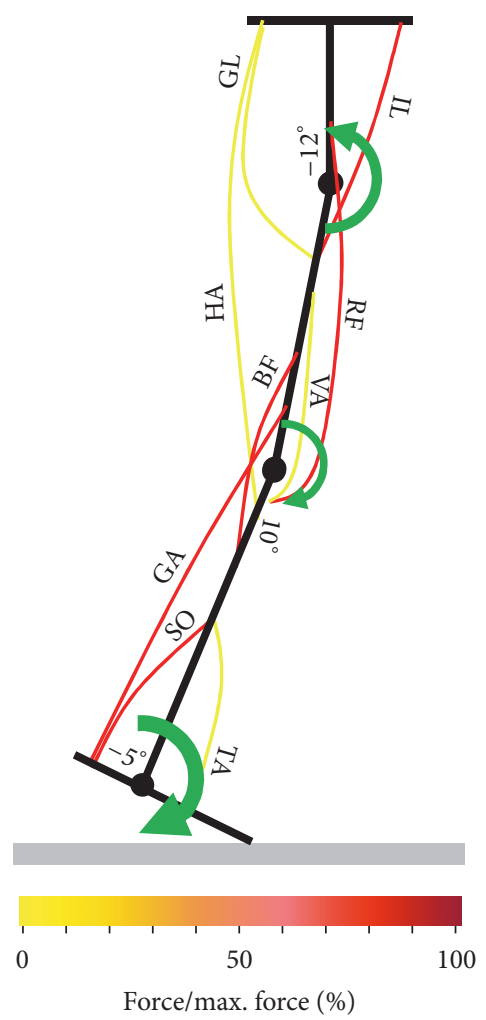

(c) Forefoot rocker $(60 \%)$

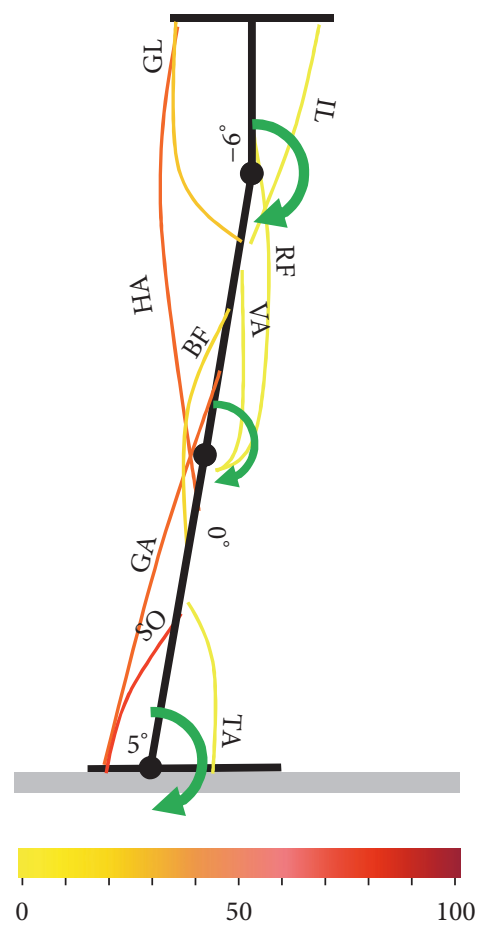

Force/max. force (\%)

(b) Ankle rocker (40\%)

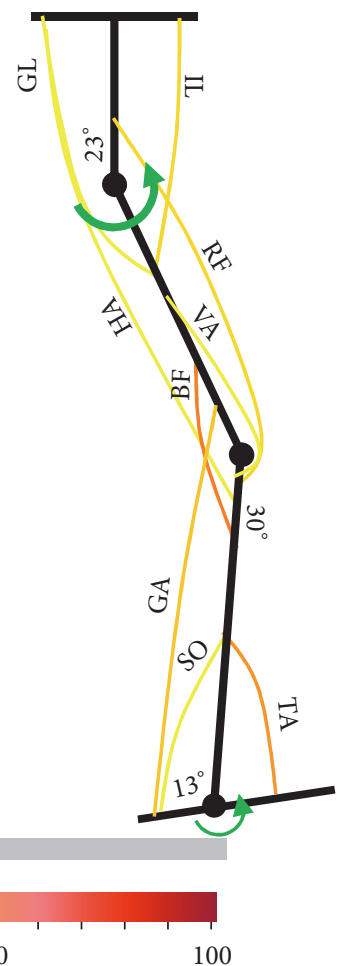

Force/max. force (\%)

(d) Floor clearance $(85 \%)$

FIGURE 10: Visualized experimental results at the four important gait functions. The posture of the segments was based on the measured joint angles, the green arrows represent directions and relative magnitudes of joint torques estimated by the inverse dynamics, and intensity of the red color represents the usage of each muscle determined by the static optimization. 

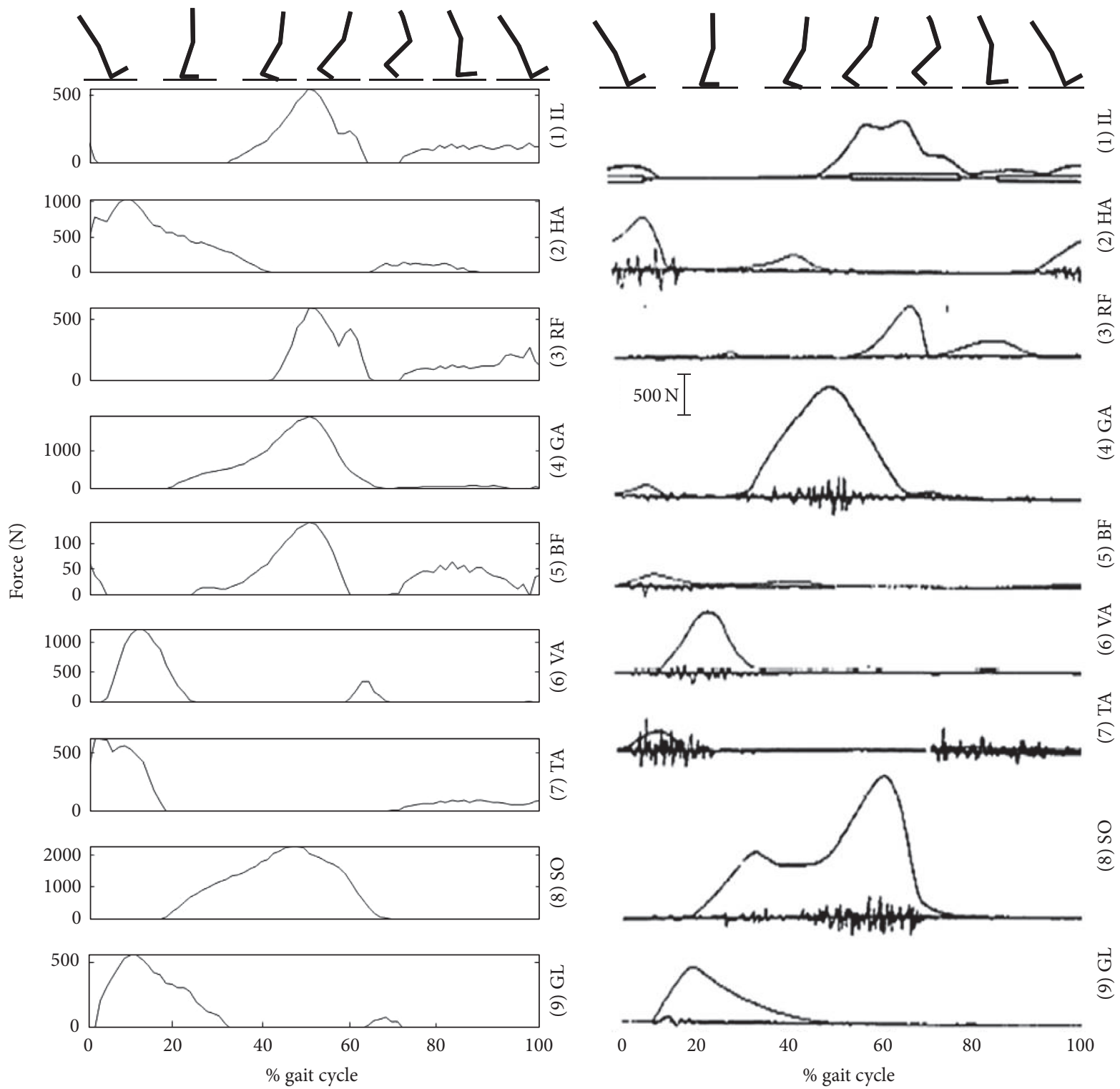

(a)

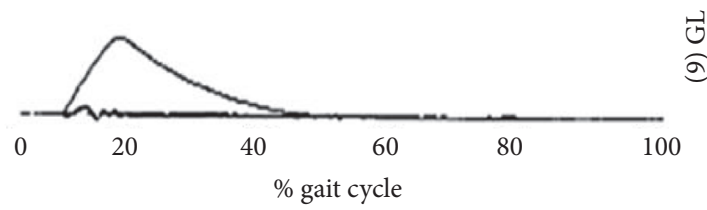

(b)

FIGURE 11: Comparison of the estimated muscular forces: (a) proposed method (b) model-based estimation with camera-based motion capture system and a force plate.

procedures, and the algorithm involved a simple musculoskeletal model with lower computation cost. By comparing with the camera-based motion capture system with a force plate, the performance of the proposed sensor system was verified experimentally, and the estimated muscular forces were interpreted with respect to the four important gait functions and were compared with the previous research with EMG signals. As a result, it was found that activation patterns of the muscles were estimated successfully by the proposed method.

Note that there are subject-specific parameters such as muscle origin and insertion positions and physiological cross-sectional areas, which can not be measured directly.
Because parameter values from the cadaveric experiment were adopted instead, a certain level of modeling uncertainties would affect the estimated results. In addition, the estimated muscular efforts would be feasible under the condition that the human body recruits and activates muscles in the optimal way. It is impossible for the proposed model to detect abnormal muscle functions such as activation patterns of disabled muscles. These are inherent limitation of the inverse dynamics-based static optimization. In this work, the objective was estimating action of the muscular system to realize the movement measured by the wearable sensor system rather than finding true value of muscular forces. However, it would be possible to suggest a special model to 
estimate muscular forces of the patients with musculoskeletal disorder such as stroke survivors with additional parameters or constraints.

\section{Appendix}

The equality constraint in static optimization was specified as follows:

$$
X=\left[\begin{array}{c}
f_{\mathrm{IL}} \\
f_{\mathrm{HA}} \\
f_{\mathrm{RF}} \\
f_{\mathrm{GA}} \\
f_{\mathrm{BF}} \\
f_{\mathrm{VA}} \\
f_{\mathrm{TA}} \\
f_{\mathrm{SO}} \\
f_{\mathrm{GL}}
\end{array}\right]
$$$$
A=\left[\begin{array}{ccc}
0 & 0 & r_{\mathrm{IL}} \\
0 & -r_{\mathrm{HA}, k} & -r_{\mathrm{HA}, h} \\
0 & r_{\mathrm{RF}, k} & r_{\mathrm{RF}, h} \\
-r_{\mathrm{GA}, a} & -r_{\mathrm{GA}, k} & 0 \\
0 & -r_{\mathrm{BF}} & 0 \\
0 & r_{\mathrm{VA}} & 0 \\
r_{\mathrm{TA}} & 0 & 0 \\
-r_{\mathrm{SO}} & 0 & 0 \\
0 & 0 & -r_{\mathrm{GL}}
\end{array}\right]^{T}
$$

$$
B=\left[\begin{array}{lll}
M_{a} & M_{k} & M_{h}
\end{array}\right]^{T},
$$

where $X$ refers to a vector of muscular forces, $r$ means the moment arm of the muscle with respect to the corresponding joint in the subscript, $a, k$, and $h$ mean the ankle, knee, and hip joints, respectively, and $M$ is the joint moment calculated by the inverse dynamics.

\section{Disclosure}

Preliminary versions of this paper were presented at the IEEE/ASME International Conference on Advanced Intelligent Mechatronics (AIM) 2015.

\section{Conflicts of Interest}

The authors declare that they have no conflicts of interest.

\section{Acknowledgments}

This work was supported by the 2016 Research Fund (1.160005.01) of UNIST (Ulsan National Institute of Science and Technology) and Basic Science Research Program through the National Research Foundation of Korea (NRF) funded by the Ministry of Science, ICT \& Future Planning (NRF-2015R1C1A1A01053763).

\section{References}

[1] V. Zatsiorsky, Kinetics of Human Motion, Human Kinetics, 2002.

[2] S. Jenkins, Sports Science Handbook, Sunningdale Publications, 2001.

[3] R. Teasell, "Stroke recovery and rehabilitation," Stroke, vol. 34, no. 2, pp. 365-366, 2003.

[4] H. M. Herr and R. D. Kornbluh, "In New horizons for orthotic and prosthetic technology: artificial muscle for ambulation," in Proceedings of Smart Structures and Materials 2004: Electroactive Polymer Actuators and Devices (EAPAD), vol. 5385 of Proceedings of SPIE, July 2004.

[5] A. T. Asbeck, R. J. Dyer, A. F. Larusson, and C. J. Walsh, "Biologically-inspired soft exosuit," in Proceedings of the IEEE 13th International Conference on Rehabilitation Robotics (ICORR '13), pp. 1-8, June 2013.

[6] Y.-L. Park, B.-R. Chen, D. Young et al., "Bio-inspired active soft orthotic device for ankle foot pathologies," in Proceedings of the IEEE/RSJ International Conference on Intelligent Robots and Systems (IROS '11), pp. 4488-4495, September 2011.

[7] M. Wehner, B. Quinlivan, P. M. Aubin et al., "A lightweight soft exosuit for gait assistance," in Proceedings of the IEEE International Conference on Robotics and Automation (ICRA '13), pp. 3362-3369, May 2013.

[8] V. Wank, R. Bauer, B. Walter et al., "Accelerated contractile function and improved fatigue resistance of calf muscles in newborn piglets with IUGR," American Journal of Physiology-Regulatory, vol. 278, no. 2, pp. R304-R310, 2000.

[9] C. J. De Luca, "The use of surface electromyography in biomechanics," Journal of Applied Biomechanics, vol. 13, no. 2, pp. 135$163,1997$.

[10] N. J. Cronin and G. Lichtwark, "The use of ultrasound to study muscle-tendon function in human posture and locomotion," Gait and Posture, vol. 37, no. 3, pp. 305-312, 2013.

[11] O. Amft, H. Junker, P. Lukowicz, G. Tröster, and C. Schuster, "Sensing muscle activities with body-worn sensors," in Proceedings of the International Workshop on Wearable and Implantable Body Sensor Networks (BSN '06), pp. 138-141, April 2006.

[12] S. L. Delp, F. C. Anderson, A. S. Arnold et al., "OpenSim: opensource software to create and analyze dynamic simulations of movement," IEEE Transactions on Biomedical Engineering, vol. 54, no. 11, pp. 1940-1950, 2007.

[13] D. G. Thelen, F. C. Anderson, and S. L. Delp, "Generating dynamic simulations of movement using computed muscle control," Journal of Biomechanics, vol. 36, no. 3, pp. 321-328, 2003.

[14] J. J. Collins, "The redundant nature of locomotor optimization laws," Journal of Biomechanics, vol. 28, no. 3, pp. 251-267, 1995.

[15] R. D. Crowninshield, R. C. Johnston, J. G. Andrews, and R. A. Brand, "A biomechanical investigation of the human hip," Journal of Biomechanics, vol. 11, no. 1-2, pp. 75-85, 1978.

[16] R. D. Crowninshield and R. A. Brand, "A physiologically based criterion of muscle force prediction in locomotion," Journal of Biomechanics, vol. 14, no. 11, pp. 793-801, 1981.

[17] U. Glitsch and W. Baumann, "The three-dimensional determination of internal loads in the lower extremity," Journal of Biomechanics, vol. 30, no. 11-12, pp. 1123-1131, 1997. 
[18] A. G. Patriarco, R. W. Mann, S. R. Simon, and J. M. Mansour, "An evaluation of the approaches of optimization models in the prediction of muscle forces during human gait," Journal of Biomechanics, vol. 14, no. 8, pp. 513-525, 1981.

[19] D. R. Pedersen, R. A. Brand, and D. T. Davy, "Pelvic muscle and acetabular contact forces during gait," Journal of Biomechanics, vol. 30, no. 9, pp. 959-965, 1997.

[20] H. Röhrle, R. Scholten, C. Sigolotto, W. Sollbach, and H. Kellner, "Joint forces in the human pelvis-leg skeleton during walking," Journal of Biomechanics, vol. 17, no. 6, pp. 409-424, 1984.

[21] A. Seireg and R. J. Arvikar, "The prediction of muscular load sharing and joint forces in the lower extremities during walking," Journal of Biomechanics, vol. 8, no. 2, pp. 89-102, 1975.

[22] A. Schultz, K. Haderspeck, D. Warwick, and D. Portillo, "Use of lumbar trunk muscles in isometric performance of mechanically complex standing tasks," Journal of Orthopaedic Research, vol. 1, no. 1, pp. 77-91, 1983.

[23] J. C. Bean, D. B. Chaffin, and A. B. Schultz, "Biomechanical model calculation of muscle contraction forces: a double linear programming method," Journal of Biomechanics, vol. 21, no. 1, pp. 59-66, 1988.

[24] R. E. Hughes, D. B. Chaffin, S. A. Lavender, and G. B. J. Andersson, "Evaluation of muscle force prediction models of the lumbar trunk using surface electromyography," Journal of Orthopaedic Research, vol. 12, no. 5, pp. 689-698, 1994.

[25] W. Z. Kong, V. K. Goel, and L. G. Gilbertson, "Prediction of biomechanical parameters in the lumbar spine during static sagittal plane lifting," Journal of Biomechanical Engineering, vol. 120, no. 2, pp. 273-280, 1998.

[26] M. A. Nussbaum and D. B. Chaffin, "Lumbar muscle force estimation using a subject-invariant 5-parameter EMG-based model," Journal of Biomechanics, vol. 31, no. 7, pp. 667-672, 1998.

[27] S. H. M. Brown and J. R. Potvin, "Constraining spine stability levels in an optimization model leads to the prediction of trunk muscle cocontraction and improved spine compression force estimates," Journal of Biomechanics, vol. 38, no. 4, pp. 745-754, 2005.

[28] N. Brook, J. Mizrahi, M. Shoham, and J. Dayan, "A biomechanical model of index finger dynamics," Medical Engineering and Physics, vol. 17, no. 1, pp. 54-63, 1995.

[29] D. D. Penrod, D. T. Davy, and D. P. Singh, "An optimization approach to tendon force analysis," Journal of Biomechanics, vol. 7, no. 2, pp. 123-129, 1974.

[30] R. Raikova and H. Aladjov, "The influence of the way the muscle force is modeled on the predicted results obtained by solving indeterminate problems for a fast elbow flexion," Computer Methods in Biomechanics and Biomedical Engineering, vol. 6, no. 3, pp. 181-196, 2003.

[31] J. Pierce and G. Li, "Muscle forces predicted using optimization methods are coordinate system dependent," Journal of Biomechanics, vol. 38, pp. 695-702, 2005.

[32] R. E. Hughes, M. G. Rock, and K.-N. An, "Identification of optimal strategies for increasing whole arm strength using Karush-Kuhn-Tucker multipliers," Clinical Biomechanics, vol. 14, no. 9, pp. 628-634, 1999.

[33] H.-T. Lin, F.-C. Su, H.-W. Wu, and K.-N. An, "Muscle forces analysis in the shoulder mechanism during wheelchair propulsion," Journal of Engineering in Medicine, vol. 218, pp. 213-221, 2004.

[34] S. Van Drongelen, L. H. Van Der Woude, T. W. Janssen, E. L. Angenot, E. K. Chadwick, and D. H. Veeger, "Glenohumeral contact forces and muscle forces evaluated in wheelchairrelated activities of daily living in able-bodied subjects versus subjects with paraplegia and tetraplegia," Archives of Physical Medicine and Rehabilitation, vol. 86, no. 7, pp. 1434-1440, 2005.

[35] D. Vlasic, R. Adelsberger, G. Vannucci et al., In Practical Motion Capture in Everyday Surroundings, ACM Transactions on Graphics (TOG), 2007.

[36] T. Cloete and C. Scheffer, "Benchmarking of a full-body inertial motion capture system for clinical gait analysis," in Proceedings of the 30th Annual International Conference of the IEEE Engineering in Medicine and Biology Society (EMBS '08), pp. 45794582, Vancouver, Canada, August 2008.

[37] M. Brodie, A. Walmsley, and W. Page, "Fusion motion capture: a prototype system using inertial measurement units and gps for the biomechanical analysis of ski racing," Sports Technology, vol. 1, pp. 17-28, 2008.

[38] D. Roetenberg, H. Luinge, and P. Slycke, Xsens mvn: Full 6dof Human Motion Tracking Using Miniature Inertial Sensors, Xsens Motion Technologies BV, Tech., 2009.

[39] Y. Zheng, K.-C. Chan, and C. C. L. Wang, "Pedalvatar: an IMUbased real-time body motion capture system using foot rooted kinematic model," in Proceedings of the IEEE/RSJ International Conference on Intelligent Robots and Systems (IROS '14), pp. 4130-4135, September 2014.

[40] Xsens, "MVN Biomech," https://www.xsens.com/products/ mvn-biomech/.

[41] Perception Neuron, "Perception Neuron," 2015, https:// neuronmocap.com/products/perception_neuron.

[42] Synertial, "IGS-Cobra Professional Mo-Cap Solutions," 2015, http://synertial.com/products/.

[43] Meta motion, "IGS 190 Motion Capture System," 2015, http:// www.metamotion.com/gypsy/gypsy-gyro.htm.

[44] Technaid, “Motion Capture System," 2015, http://www.technaid .com/en/products/motion-capture-system.

[45] G. Cooper, I. Sheret, L. McMillian et al., "Inertial sensor-based knee flexion/extension angle estimation," Journal of Biomechanics, vol. 42, no. 16, pp. 2678-2685, 2009.

[46] T. Khurelbaatar, K. Kim, S. Lee, and Y. H. Kim, "Consistent accuracy in whole-body joint kinetics during gait using wearable inertial motion sensors and in-shoe pressure sensors," Gait and Posture, vol. 42, no. 1, pp. 65-69, 2015.

[47] J.-C. Kim, K.-S. Kim, and S. Kim, "Wearable sensor system including optical 3-axis GRF sensor for joint torque estimation in real-time gait analysis," in Proceedings of the IEEE/ASME International Conference on Advanced Intelligent Mechatronics (AIM '14), pp. 112-117, July 2014.

[48] S. J. M. Bamberg, A. Y. Benbasat, D. M. Scarborough, D. E. Krebs, and J. A. Paradiso, "Gait analysis using a shoe-integrated wireless sensor system," IEEE Transactions on Information Technology in Biomedicine, vol. 12, no. 4, pp. 413-423, 2008.

[49] N. K. Lee, R. S. Goonetilleke, Y. S. Cheung, and G. M. So, "A flexible encapsulated MEMS pressure sensor system for biomechanical applications," Microsystem Technologies, vol. 7, no. 2, pp. 55-62, 2001.

[50] S. Kim, K. Ro, and J. Bae, "Real-time estimation of individual muscular forces of the lower limb using wearable sensors," in Proceedings of the IEEE/ASME International Conference on Advanced Intelligent Mechatronics (AIM '15), pp. 432-436, Pusan, South Korea, July 2015.

[51] Withrobot, “myahrs+," 2017, http://www.withrobot.com/ myahrs_en/. 
[52] J. Bae, K. Kong, N. Byl, and M. Tomizuka, "A mobile gait monitoring system for abnormal gait diagnosis and rehabilitation: a pilot study for Parkinson disease patients," Journal of Biomechanical Engineering, vol. 133, no. 4, p. 041005, 2011.

[53] J. Bae and M. Tomizuka, "A tele-monitoring system for gait rehabilitation with an inertial measurement unit and a shoetype ground reaction force sensor," Mechatronics, vol. 23, no. 6, pp. 646-651, 2013.

[54] J. Perry and J. Davids, "Gait analysis: normal and pathological function," Journal of Pediatric Orthopaedics, vol. 12, p. 815, 1992.

[55] G. Li, T. Liu, L. Gu, Y. Inoue, H. Ning, and M. Han, "Wearable gait analysis system for ambulatory measurement of kinematics and kinetics," in Proceedings of the 13th IEEE SENSORS Conference (SENSORS '14), pp. 1316-1319, November 2014.

[56] J. Bae, K. Kong, and M. Tomizuka, "Real-time estimation of lower extremity joint torques in normal gait," Robot Control, vol. 9, pp. 443-448, 2009.

[57] K. Kong and M. Tomizuka, "A gait monitoring system based on air pressure sensors embedded in a shoe," IEEE/ASME Transactions on Mechatronics, vol. 14, no. 3, pp. 358-370, 2009.

[58] B. Bresler and J. Frankel, "The forces and moments in the leg during level walking," Transactions of the American Society of Mechanical Engineers, vol. 72, pp. 25-35, 1950.

[59] D. A. Winter, Biomechanics and Motor Control of Human Movement, John Wiley \& Sons, 2009.

[60] D. Miller and R. Nelson, Biomechanics of Sports, Lea and Febiger, 1973.

[61] Dempster, Patterns of Human Motion, Prentice-Hall, 1971.

[62] S. Heintz, "Muscular forces from static optimization," 2006.

[63] A. Pedotti, V. V. Krishnan, and L. Stark, "Optimization of muscle-force sequencing in human locomotion," Mathematical Biosciences, vol. 38, no. 1-2, pp. 57-76, 1978.

[64] M. G. Hoy, F. E. Zajac, and M. E. Gordon, "A musculoskeletal model of the human lower extremity: the effect of muscle, tendon, and moment arm on the moment-angle relationship of musculotendon actuators at the hip, knee, and ankle," Journal of Biomechanics, vol. 23, no. 2, pp. 157-169, 1990.

[65] R. A. Brand, R. D. Crowninshield, C. E. Wittstock, D. R. Pedersen, C. R. Clark, and F. M. Van Krieken, "Model of lower extremity muscular anatomy," Journal of Biomechanical Engineering, vol. 104, pp. 304-310, 1982.

[66] R. J. Maughan, J. S. Watson, and J. Weir, "Strength and cross-sectional area of human skeletal muscle," The Journal of Physiology, vol. 338, no. 1, pp. 37-49, 1983.

[67] E. M. Arnold, S. R. Ward, R. L. Lieber, and S. L. Delp, "A model of the lower limb for analysis of human movement," Annals of Biomedical Engineering, vol. 38, no. 2, pp. 269-279, 2010.

[68] Motion Analysis Corp, "Kestrel digital realtime system," 2017, http://www.motionanalysis.com/html/movement/kestrel.html.

[69] Kistler, "Multicomponent force plate," 2017, https://www.kistler .com $/$ ?type $=669 \&$ fid $=60693 \&$.

[70] A. Cappozzo, T. Leo, and A. Pedotti, "A general computing method for the analysis of human locomotion," Journal of Biomechanics, vol. 8, no. 5, pp. 307-320, 1975. 


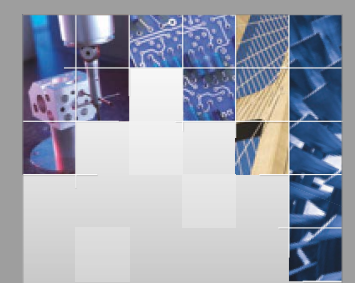

\section{Enfincering}
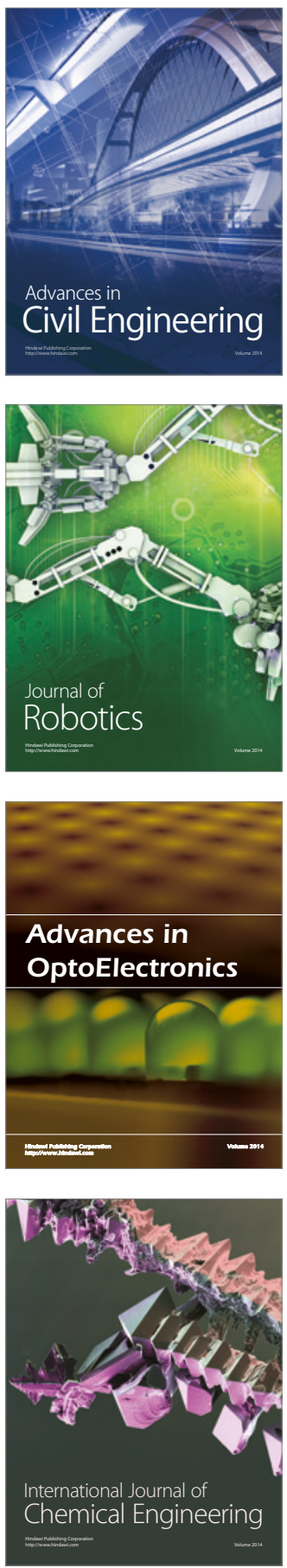

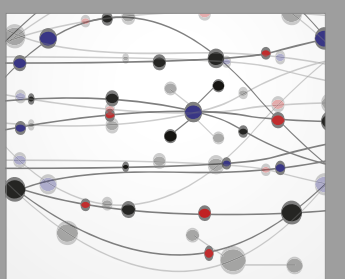

The Scientific World Journal

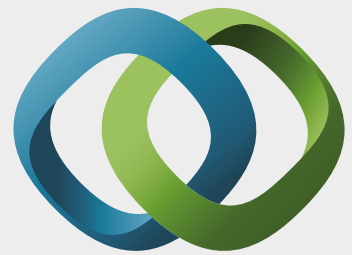

\section{Hindawi}

Submit your manuscripts at

https://www.hindawi.com
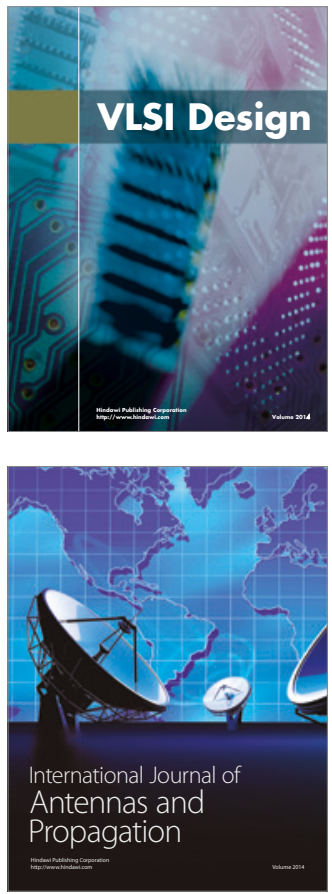

\section{Rotating}

Machinery
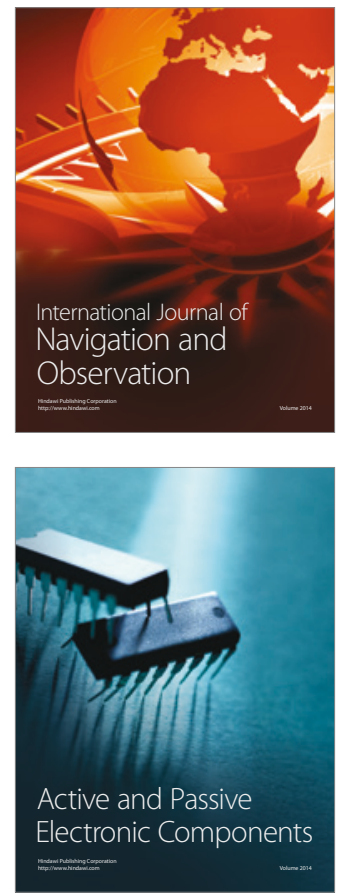
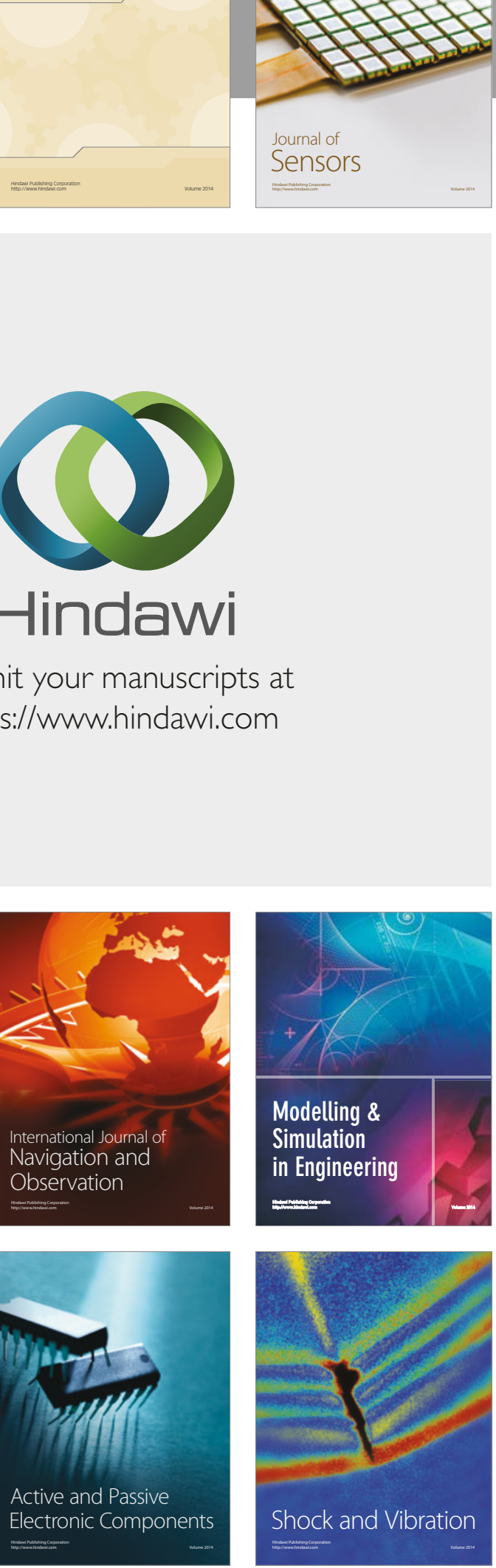
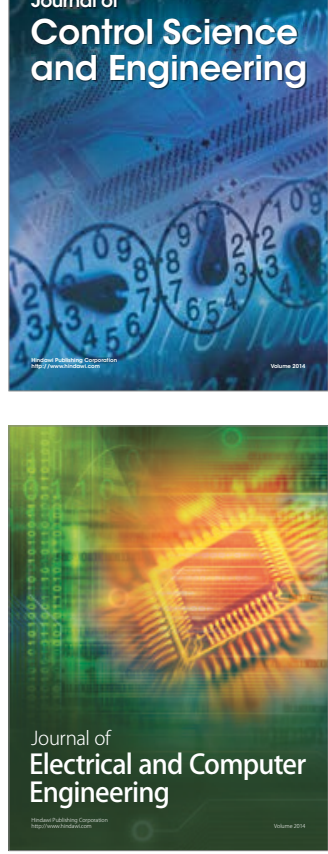

Distributed

Journal of

Control Science

and Engineering
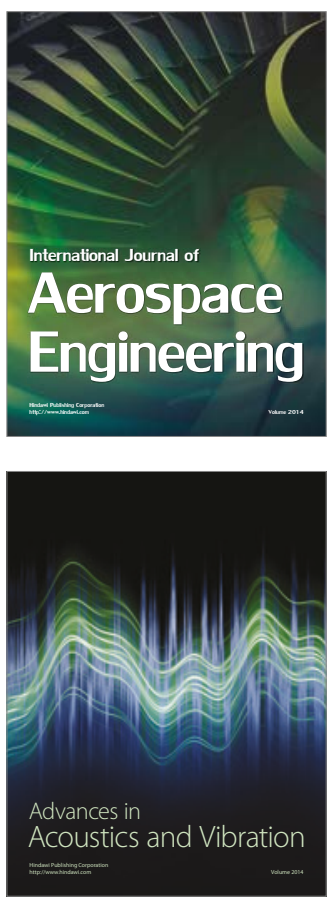

Sensor Networks 